\title{
Cerebral hemorrhage in a dog with immune-mediated hemolytic anemia and concurrent thrombocytopenia - a case report
}

\author{
EKREM ÇAĞATAY ÇOLAKOĞLU1', ALI EVREN HAYDARDEDEOĞLU², \\ HADI ALIHOSSEINI ${ }^{3}$, ECE AYDEMIR ${ }^{1}$
}

\author{
${ }^{1}$ Department of Internal Medicine, Faculty of Veterinary Medicine, Ankara University, 06110 Turkey \\ ${ }^{2}$ Department of Internal Medicine, Faculty of Veterinary Medicine, Aksaray University, 68100, Turkey \\ ${ }^{3}$ Therapist Veterinary Medical Center, İstanbul, 34337, Turkey
}

Çolakoğlu E. Ç., Haydardedeoğlu A. E., Alihosseini H., Aydemir E.

Cerebral hemorrhage in a dog with immune-mediated hemolytic anemia and concurrent thrombocytopenia - a case report

\section{Summary}

The aim of this case report is to point out the neurological signs, long term management of the case and MRI lesions suggestive of cerebral haemorrhage in a dog with IMHA and concurrent thrombocytopenia. A 3.5-year-old intact female Cocker Spaniel dog was examined for anorexia, weakness, exercise intolerance and two collapse episodes. Based on the diagnostic procedures primary immune mediated hemolytic anaemia concurrent with severe thrombocytopenia were confirmed. The dog remained stable with prednisolone and fluid therapy for 6 weeks. The dog was later referred to emergency service with hemianopia, head tilt and epilepsy-like signs. Brain MRI lesions showed central line deviation, oedema and cerebral haemorrhage. Post-treatment MRI also provided clear evidence of resolution the cerebral haemorrhage. The dog was asymptomatic for 6 months after splenectomy. To the best our knowledge, this is the first fully documented report of cerebral haemorrhage in a dog with immune mediated hemolytic anaemia and thrombocytopenia.

Keywords: cerebral haemorrhage, dog, hemolytic anemia, thrombocytopenia

Immune-mediated hemolytic anemia (IMHA) characterized by activation of the complement system and production of autoantibodies resulting in erythrocyte destruction is one of the most common hematological problems among dogs $(7,23)$. IMHA diagnosis is a rule-out diagnosis. When an underlying constructive reason such as neoplasia, chronic infectious diseases, exposure to medication, toxicity and previous vaccinations could not be found, IMHA may develop with primary origin in $75 \%$ of dogs $(6,8)$. Concurrent thrombocytopenia defined as production of antibodies against platelet surface antigens is also reported in dogs with $\operatorname{IMHA}(4,14,21,24)$.

Many studies describe high mortality rates during the first 2-3 weeks of treatment and due to complications associated with IMHA and coagulopathy $(3,18,19)$. Platelet destruction and concurrent primary hemostasis deficiencies may lead to an increase in tendencies for hemorrhage such as cutaneous petechia and ecchymoses, melena, hyphema, retinal hemorrhage, epistaxis and hematuria $(2,5,25)$. Veterinary literature on coagulopathy-related cerebral alterations and associated neurological symptoms is limited $(10,24,25)$.

This case report describes a dog with cerebral hemorrhage secondary to immune-mediated hemolytic anemia and concurrent thrombocytopenia.

\section{Case history}

A 3.5-year-old intact female Cocker Spaniel dog weighing $10.2 \mathrm{~kg}$ was evaluated by an animal hospital for anorexia, weakness, exercise intolerance and two collapse episodes witnessed by the owners. The dog had undergone routine antiparasitic therapy and vaccination against rabies, distemper, parainfluenza, hepatitis, parvoviral enteritis and leptospirosis. It was also fed with commercial dry food. At presentation, the dog was lethargic and tachypneic (respiratory rate $=47 \mathrm{breath} / \mathrm{pm}$ ). Physical examination revealed mucosal pallor with a body temperature of $37.1^{\circ} \mathrm{C}$, abdominal distress and tachycardia (Heart rate $=163 \mathrm{bpm}$ ). Grade I-II/VI left apical systolic heart murmurs were auscultated. 
The dog was hospitalized with supplemental oxygen delivery.

Routine blood analyses, urinalysis, ultrasonography, thoracic graphies, and echocardiography were performed during hospitalization. No remarkable changes were observed by echocardiography or thoracic images. Abdominal ultrasonography revealed splenomegaly. Complete blood count, coagulation variables (PT, aPTT, D-dimer) and serum profiles are shown in Table 1. Primary immune-mediated hemolytic anemia (IMHA) concurrent with severe thrombocytopenia were strongly confirmed by diagnostic procedures including spherocytes on peripheral blood smears, a positive Coombs' test, true agglutination persisting after dilution in phosphatebuffered saline, as well as bilirubinuria and hemoglobinuria in urine dipstick tests. Serologies for Ehrlichia canis, Babesia canis, Anaplasma phagocytophilum, Borrelia burgdorferi, Leishmania infantum, Rickettsia rickettsia, and Mycoplasma canis obtained with an indirect fluorescent antibody test (IFAT) were all negative.

The dog initially could not tolerate p.o. treatment with prednisolone. Medication was initiated with fluid therapy $(0.9 \% \mathrm{NaCl}$ solution and fluids including electrolytes, amino acids and dextrose) and injectable dexamethasone $(0.4 \mathrm{mg} /$ $\mathrm{kg}$ /day i.v.) for 7 days. After 7 days following the therapy in hospital, prednisolone at an initial p.o. dosage of $2 \mathrm{mg} / \mathrm{kg} /$ day with famotidine (p.o. $1 \mathrm{mg} / \mathrm{kg} /$ day) were administered at home for 2 weeks. The dog remained stable for the next 6 weeks with a reduced dosage of prednisolone $(1.5 \mathrm{mg} / \mathrm{kg} / \mathrm{day})$. Two months later the dog was referred to emergency service with hemianopia, head tilt and seizure. Fundus imaging was normal. CBC (complete blood count) revealed severe thrombocytopenia and anemia (Tab. 2). Phenobarbital (2 mg/kg p.o. q $12 \mathrm{~h}$ ), diazepam ( $1 \mathrm{mg} / \mathrm{kg}$ i.v. q $8 \mathrm{~h}$ ) and whole blood transfusion (with a DEA 1.1 + blood product) initiated before MRI. Brain MRI was performed with high field 1.5 T Magnetom (Siemens Vision Plus, New Jersey, USA) under general anaesthesia. Both exams included T1W TSE, T2W TSE, contrast enhanced (Gadobutrol) T1W and FLAIR sequences respectively (Fig. 1). MRI features and contrast enhancements were
Tab. 1. Initial blood analysis

\begin{tabular}{|c|c|c|c|c|c|}
\hline \multicolumn{2}{|c|}{ CBC Results } & \multirow{2}{*}{$\begin{array}{l}\text { References } \\
6.00-17.00\end{array}$} & \multicolumn{2}{|c|}{ Biochemical Profiles } & \multirow{2}{*}{$\begin{array}{c}\text { References } \\
65-118\end{array}$} \\
\hline WBC $\left(10^{9} / \mathrm{I}\right)$ & 7.70 & & Glucose (mg/dl) & 73 & \\
\hline LYM $\left(10^{9} / 1\right)$ & 1.17 & $1.0-4.80$ & Urea (mg/dl) & 28.6 & $15-60$ \\
\hline MONO $\left(1^{9} / l\right)$ & 0.59 & $0.2-1.5$ & Creatinine (mg/dl) & 0.72 & $0.5-1.5$ \\
\hline NEUT $\left(10^{9} / 1\right)$ & 5.84 & $3.00-12.00$ & Total Protein (g/dl) & 7.1 & $5.4-7.1$ \\
\hline EOS $\left(10^{9} / l\right)$ & 0.1 & $0.1-19$ & Albumine (g/dl) & 3.6 & $3.1-4.0$ \\
\hline LYM (\%) & 15.2 & $12-30$ & Total Bilirubin (mg/dl) & 0.42 & $0.1-0.3$ \\
\hline MONO (\%) & 7.66 & $3-10$ & Direct Bilirubin (mg/dl) & 0.37 & \\
\hline NEUT (\%) & 75.8 & $62-87$ & ALP (IU/L) & 172.0 & $20-156$ \\
\hline EOS (\%) & 1.29 & $0.1-19$ & ALT (IU/L) & 105.7 & $21-102$ \\
\hline $\operatorname{RBC}\left(10^{12} / \mathrm{I}\right)$ & 3.30 & $5.5-8.5$ & AST (IU/L) & 41.6 & $23-66$ \\
\hline HGB (g/dl) & 8.90 & $12-18$ & GGT (IU/L) & 6.0 & $6-28$ \\
\hline HCT (\%) & 26.0 & $37-55$ & Phosphore (mg/dl) & 3.1 & $2.9-6.2$ \\
\hline MCV (fl) & 79.0 & $60-77$ & Calcium (mg/dl) & 9.5 & $9-11.3$ \\
\hline MCH (pg) & 26.9 & $19.5-24.5$ & Sodium (mmol/l) & 145 & $140-154$ \\
\hline MCHC (g/dl) & 34.1 & $31-34$ & Potassium (mmol/l) & 3.9 & $3.8-5.6$ \\
\hline RDWc (\%) & 15.6 & & \multicolumn{2}{|c|}{ Coagulation Variables } & References \\
\hline PLT (109/l) & 38.0 & $200-500$ & PT (seconds) & 7.9 & 6.2-7.7 \\
\hline РCT (\%) & 0.04 & & aPTT (seconds) & 19.4 & $9.8-14.6$ \\
\hline MPV (fl) & 9.60 & $3.9-11$ & D-dimer $(\mu \mathrm{g} / \mathrm{ml})$ & 0.53 & $0.01-0.34$ \\
\hline PDWc (\%) & 34.5 & & & & \\
\hline
\end{tabular}

Explanations: WBC - White blood cell; LYM - Lymphocyte; MONO - Monocyte; NEUT - Neutrophile; EOS - Eosinophile; RBC - Red blood cell; HGB - Hemoglobin; HCT - Hematocrit; MCV - Mean corpuscular volume; MCH - Mean corpuscular hemoglobin; MCHC - Mean corpuscular hemoglobin concentration; RDW - Red blood cell distribution width; PLT - Platelet; PCT - Plateletcrit; MPV - Mean platelet volume; PDW - Platelet distribution width; PT - prothrombin time; aPTT - Activated Partial Thromboplastin Time

Tab. 2. Follow up blood analysis

\begin{tabular}{|c|c|c|c|c|c|}
\hline Variables & $\begin{array}{l}7 \text { days after } \\
\text { dexamethasone } \\
\text { and fluid therapy }\end{array}$ & $\begin{array}{c}14 \text { days after } \\
\text { prednisolone } \\
\text { therapy }\end{array}$ & $\begin{array}{l}2 \text { months } \\
\text { after the first } \\
\text { evaluation }\end{array}$ & $\begin{array}{l}6 \text { days after } \\
\text { splenectomy }\end{array}$ & References \\
\hline WBC $\left(10^{9} / I\right)$ & 9.43 & 10.1 & 16.12 & 14.28 & $6.00-17.00$ \\
\hline LYM (109/l) & 2.04 & 1.15 & 1.35 & 2.86 & $1.0-4.80$ \\
\hline MONO (109/l) & 0.46 & 0.44 & 1.25 & 0.40 & $0.2-1.5$ \\
\hline NEUT $\left(10^{9} / I\right)$ & 6.94 & 8.45 & 13.41 & 10.82 & $3.00-12.00$ \\
\hline EOS $\left(10^{9} / l\right)$ & 0.1 & 0.06 & 0.1 & 0.2 & $0.1-19$ \\
\hline $\operatorname{RBC}\left(10^{12} / \mathrm{l}\right)$ & 4.61 & 5.32 & 4.61 & 4.80 & $5.5-8.5$ \\
\hline HGB (g/dl) & 12.5 & 13.6 & 11 & 11.6 & $12-18$ \\
\hline HCT (\%) & 36.29 & 37.01 & 32.12 & 35.11 & $37-55$ \\
\hline MCV (fl) & 79 & 76 & 70 & 73 & $60-77$ \\
\hline MCH (pg) & 27.1 & 25.5 & 23.9 & 24.2 & $19.5-24.5$ \\
\hline MCHC (g/dl) & 34.4 & 33.6 & 34.4 & 33.1 & $31-34$ \\
\hline RDWc (\%) & 17.2 & 16.8 & 17.9 & 18.4 & \\
\hline PLT (10/l) & 122 & 252 & 19 & 814 & $200-500$ \\
\hline РCT (\%) & 0.16 & 0.26 & 0.02 & 0.8 & \\
\hline MPV (fl) & 12.8 & 10.4 & 5.4 & 9.8 & $3.9-11$ \\
\hline PDWc (\%) & 44.8 & 38.1 & 31.2 & 38.1 & - \\
\hline Bilirubin (mg/dl) & 0.31 & 0.35 & - & 0.26 & $0.1-0.3$ \\
\hline D-dimer $(\mu \mathrm{g} / \mathrm{ml})$ & - & - & 0.32 & - & $0.01-0.34$ \\
\hline
\end{tabular}

Explanations: as in Tab. 1 


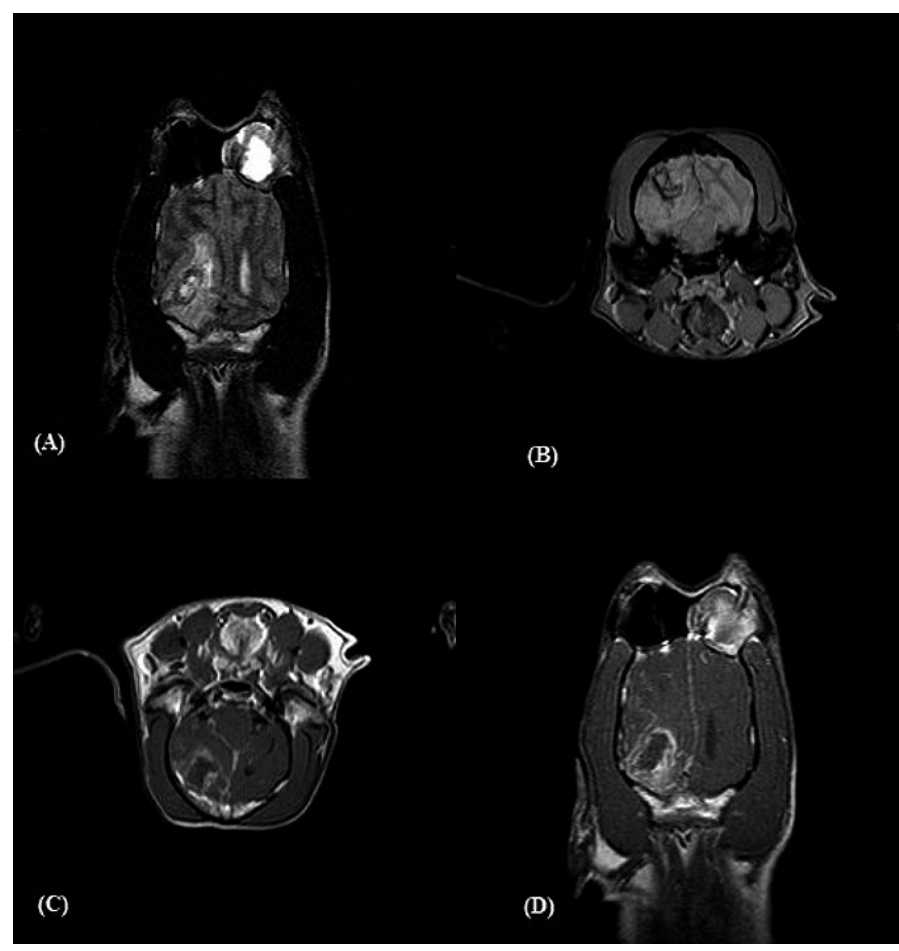

Fig. 1. Pre-treatment MRI findings of cerebral hemorrhage. Central line deviation to the left is obvious for both coroner and axial views (mass effect). Incidental finding of increased intensity in the left frontal sinus suggests fluid accumulation related to left frontal sinusitis. (A) T2W images shows a large lesion surrounded with a larger hyperintense area. (B) FLAIR sequence images indicate a more demarcated lesion view at the right temporo-parietal area. T2 and FLAIR hyperintensity around the lesions suggest oedema. $(C, D)$ The contrast enhancement in $\mathrm{T} 1 \mathrm{~W}$ images was periferal to the lesion and the center of the lesion is highly hypointense

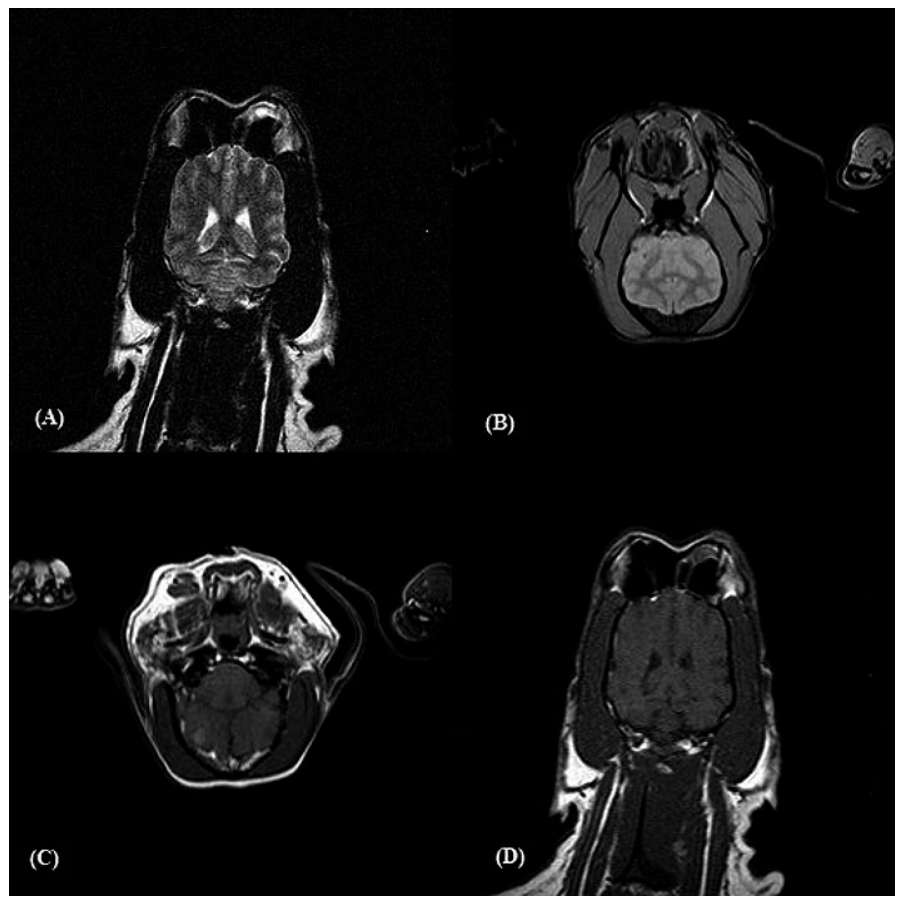

Fig. 2. (A, B) No center line shifting was present in all sequences. T2W and FLAIR sequences shows mild edema in the original lesion location. (C, D) No contrast enhancement detected in T1W TSE sequences. Both ventricles are visible and symmetrical compared to the prior state two weeks earlier considered as a large area of cerebral bleeding. Inflammatory and neoplastic lesions were considered less likely. Cerebrospinal culture and cytology were also not remarkable.

On the following day human intravenous immunoglobulin - hIVIG $(0.5 \mathrm{~g} / \mathrm{kg}$ single infusion with prescribed volume of sterile water) was added to the treatment with phenobarbital and prednisolone. Splenectomy was also performed. Brain MRI following the treatment for 6 days revealed a distinct remission of the lesion (Fig. 2). The dog was asymptomatic for 6 months after the splenectomy without being on any medication.

\section{Results and discussion}

Immune-mediated hemolytic anemia (IMHA) and concurrent thrombocytopenia are hematologic problems that may show severe clinical symptoms $(4,25)$. Concurrent development of IMHA and immune-mediated thrombocytopenia is defined as Evans syndrome in humans. A few cases of Evans syndrome have also been reported in $\operatorname{dogs}(4,11)$. It is possible to diagnose anti-platelet antibodies with some methods including flow cytometry, Elisa and immunoradiometry (22). We prefer not to define the case here as Evans syndrome due to difficulty in detecting immune mediated platelet destruction, although the case also has concurrent severe thrombocytopenia.

Cerebrovascular diseases refer to any abnormalities resulting in pathologic processes such as thrombosis, embolism or haemorrhage (26). Blood infiltrated in subarachnoid space or brain parenchyma as a result of intracranial bleeding may create neuronal damage and cause disorientation due to stress on peripheral tissue (1). Edema develops around the bleeding area after a while, and ischemia is triggered since brain tissue remains under stress caused by bleeding. Depending on the severity of hemorrhage, a mass effect with a midline shift, cerebral blood flow alteration, an increase in intracranial pressure or obstructive hydrocephalus may appear as well (1). In the case presented here, clinical signs including hemianopia, head tilt and seizure, and MRI findings suggesting edema around the lesions were consistent with pathophysiological results of the intracranial bleeding. Yet aetiology cannot be determined in many cases. Constructive reasons such as hypertension, kidney and heart diseases, neoplasia and metastasis may lead to cerebrovascular problems (9). We observed no remarkable changes in diagnostic applications including cerebrospinal culture and cytology in the dog.

Coagulopathies are identified in dogs with IMHA and immune-mediated thrombocytopenia (4). In IMHA patients, a decreased thrombocyte count may be associated with increased thrombocyte consumption due to disseminated intravascular coagulation (DIC) or the increased destruction due to immune mechanisms (4). In a study, thrombocytopenia was predominant in the majority of dogs with IMHA and the mortality rate was 
higher in the dogs compared to healthy ones. Authors also revealed the increased risk of hypercoagulability in dogs with concurrent thrombocytopenia $(4,17)$. It is very difficult to distinguish the patients with decreased platelet numbers due to Evans syndrome from those with thrombocytopenia. Prolonged prothrombin and partial thromboplastin time and increased D-dimer levels may appear in the presence of DIC as well (2). In the initial blood analyses of the case presented here, thrombocytopenia was associated with prolonged PT, aPTT and increased D-dimer level. Data from literature also support that dogs with primary IMHA at the time of first diagnosis were in a hypercoagulability state $(4,17)$. Even though coagulation deformities and bleeding may develop secondary DIC development, the dog had normal D-dimer level when it was referred to the emergency clinic with hemianopia, head tilt and seizure. Not knowing the concentrations of fibrin degradation products and fibrinogen and also normal D-dimer levels prevented us from fully excluding the possibility of platelet consumption. However, severe thrombocytopenia was most likely primarily by immune mediated destruction in the dog.

In the studies of dogs with immune-mediated thrombocytopenia, an increase in the risk of spontaneous bleeding in platelet numbers below $\leq 15.0 \times 10^{9} / 1$, $<30 \times 10^{9} / 1$ and $\leq 50.0 \times 10^{9} / 1$ is mentioned (11). In another study performed with 30 dogs with immunemediated thrombocytopenia (20), platelet levels of $77 \%$ of the dogs were below $<30 \times 10^{9} / 1.83 \%$ of these dogs had other hemorrhagic findings but they did not show cerebral hemorrhage. Although cutaneous petechia and ecchymoses, retinal hemorrhage, melena, hematemesis and epistaxis were defined as the most common physical examination findings in dogs with IMHA in veterinary medicine, there is no data on the rate of intracranial hemorrhage. However, it is reported that neurological signs and blindness are likely to occur with an increased risk of bleeding (16). In the case presented here, platelet levels of the dog both in the first examination and during neurological signs are consistent with the literature $(11,20,25)$.

Thrombocytopenia increases vascular fragility, causing alteration in vascular strength, endothelial alterations and loss of endothelial backup functions in thrombocyte. This concept is strengthened by electron microscopic analyses of microvascular alterations that occurred both in animals and human patients with thrombocytopenia $(12,13)$. In thrombocytopenia cases specifically under 20000 platelet/ $\mu 1$ threshold value, interendothelial links loosen, endothelial thinning rises and extravasation is formed (15).

In conclusion, this paper reflects the neurological signs, long term management of the case and MRI lesions suggestive of cerebral hemorrhage in a dog with IMHA and concurrent thrombocytopenia.

\section{References}

1. Auer R. N., Sutherland G. R.: Primary intracerebral hemorrhage: pathophysiology. Can. J. Neurol. Sci. 2005, 32, 3-12.

2. Balch A., Mackin A.: Canine immune-mediated hemolytic anemia: pathophysiology, clinical signs, and diagnosis. Cont. Edu. Pract. Vet. 2007, 29, 217-222.

3. Burgess K., Moore A., Rand W., Cotter S. M.: Treatment of immune-mediated hemolytic anemia in dogs with cyclophosphamide. JVIM 2000, 14, 456-462.

4. Carr A. P., Panciera D. L., Kidd L.: Prognostic factors for mortality and thromboembolism in canine immune-mediated hemolytic anemia: A retrospective study of 72 cases. J. Vet. Intern. Med. 2002, 16, 504-509.

5. Chartier M.: Canine Primary (Idiopathic) Immune-Mediated Thrombocytopenia. Clinicians Brief. 2015, 15, 82-86.

6. Cotter $S$.: Autoimmune hemolytic anemia. Compendium Continuing Education for the Practicing Veterinarian 1992, 14, 93-100.

7. Day M. J.: Immune-mediated haemolytic anaemia, [in:] Day M. J., Kohn B. (eds): BSAVA Manual of Canine and Feline Haematology and Transfusion Medicine. Gloucester, UK 2012, p. 59-73.

8. Duval D., Giger U.: Vaccine-associated immune-mediated hemolytic anemia in the dog. J. Vet. Intern. Med. 1996, 10, 290-295.

9. Garosi L. S.: Cerebrovascular disease in dogs and cats. Vet. Clin. North Am. Small. Am. Pract. 2010, 40, 65-79.

10. Giannuzzi A. P., De Simone A., Ricciardi M., Gernono F.: Presumptive Ischemic Brain Infarction in a Dog with Evans' Syndrome. Case Rep. Vet. Med. 2014.

11. Goggs R., Boag A. K., Chan D. L.: Concurrent immunemediated haemolytic anaemia and severe thrombocytopenia in 21 dogs. Vet. Rec. 2008, 163, 323-327.

12. Gore I., Takada M., Austin J.: Ultrastructural basis of experimental thrombocytopenic purpura. Archp. Pathol. 1970, 90, 197-205.

13. Kitchens C. S., Pendergast J. F.: Human thrombocytopenia is associated withstructural abnormalities of the endothelium that are ameliorated by glucocorticosteroid administration. Blood 1986, 67, 203-206.

14. Klag A., Giger U., Shofer F.: Idiopathic immune-mediated hemolytic anemia in dogs: 42 cases (1986-1990). JAVMA 1993, 202, 783-788.

15. Levine D. N., Cianciolo R. E., Linder K. E., Bizikova P., Birkenheuer A. J., Brooks M. B., Salous A. K., Nordone S. K., Bellinger D. A., Marr H., Jones S. L., Fischer T. H., Deng Y., Mazepa Mi Key N. S.: Endothelial alterations in a canine model of immunethrombocytopenia. Platelets 2007, 30, 88-97.

16.Lewis D. C., Meyers K. M., Callan M. B., Bücheler J., Giger U.: Detection of platelet-bound and serum platelet-bindable antibodies for diagnosis of thrombocytopenic purpura in dogs. JAVMA 1995, 206, 47-52.

17. Mischke V. R.: Haemostatic disorders as a complication of autoimmunohaemolytic anaemia in dogs. Dtsch. Tierarztl. henschri. 1998, 105, 13-16.

18. Piek C. J., Junius G., Dekker A., Schrauwen E., Slappendel R. J., Teske E. Idiopathic immune-mediated hemolyic anemia: treatment, outcome and prognostic factors in 149 dogs. J. Vet. Intern. Med. 2008, 22, 366-373.

19. Piek C. J., Van Spil W. E., Junius G., Dekker A.: Lack of evidence of a beneficial effect of azathioprine in dogs treated with prednisolone for idiopathic immunemediated hemolytic anaemia: a retrospective cohort study. BMC Vet. Res. 2011, 7, 15-23.

20. Putsche J. C., Kohn B.: Primary immune-mediated thrombocytopenia in 30 dogs (1997-2003). JAAHA 2008, 44, 250-257.

21. Ridyard A. E., Shaw D. J., Milne E. M.: Evaluation of platelet activation in canine immune-mediated haemolytic anaemia. JSAP 2010, 51, 296-304.

22. Scott M. A., Kaiser L., Davis J. M., Kenneth B. S., Scwarts M. D.: Development of a sensitive immunoradiometric assay for detection of platelet surface associated immunoglobulins in thrombocytopenic dogs. Am. J. Vet. Res. 2002, 63, 124-129.

23. Weingart C., Thielemann D., Kohn B.: Primary immune-mediated haemolytic anaemia: a retrospective long-term study in 61 dogs. Aust. Vet. J. 2019, 97 , 483-489.

24. Weiss D. J., Brazzell J. L.: Detection of activated platelets in dogs with primary immune-mediated hemolytic anemia. J. Vet. Intern. Med. 2006, 20, 682-686.

25. Williams D. A., Maggio-Price L.: Canine idiopathic thrombocytopenia: clinical observations and long-term follow-up in 54 cases. JAVMA 1984, 185, 660-663.

26. Wonsiewicz M. J., Medina M. P., Navrozov M.: Adams and Victor's Principles of Neurology. New York, USA McGraw-Hill 2001.

Corresponding author: Assoc. Prof. Ekrem Çağatay Çolakoğlu, Department of Internal Medicine, Faculty of Veterinary Medicine, Ankara University, 06110 Ankara, Turkey; e-mail: colakoglu@ankara.edu.tr 